\title{
Study of Molecules with Multiple Redox Centers Using Differential Staircase Voltammetry at Spherical Electrodes and Microelectrodes
}

\author{
Manuela López-Tenés, Carmen Serna, Marién M. Moreno, Ángela Molina* \\ Departamento de Química Física, Universidad de Murcia, Espinardo 30100, Murcia, Spain
}

\begin{abstract}
The study of the reversible reduction/oxidation molecules containing multiple interacting or noninteracting redox centers has been carried out using the multipotential step technique Differential Staircase Voltammetry (DSCV). Methods to obtain the formal potentials and other characteristic parameters of the process are proposed for any interaction degree between the centers, when using spherical electrodes of any size (from planar to ultramicrospherical electrodes) and, in particular, the advantages of microelectrodes are discussed. The theoretical predictions have been tested with two experimental systems: ethyl viologen in acetonitrile and pyrazine in aqueous acid media, finding an excellent agreement between the theory and experiments. Therefore, the formal potentials for these systems have been evaluated using DSCV.
\end{abstract}

Keywords: Multicenter molecules; interacting and noninteracting redox centers; multipotential techniques; Differential Staircase Voltammetry; DSCV; spherical electrodes; microelectrodes.

\section{Introduction}

At present, the electrochemical behaviour of multicenter molecules plays a key role in the interpretation of the interactions between the different groups in the molecule. The study of these molecules is of enormous interest, since their applications in many different fields are being considered [1-3].

In this paper we study the reduction of a molecule containing $n$ electroactive redox centers, according to the following scheme:

\footnotetext{
* Corresponding author. E-mail address: amolina@um.es
} 


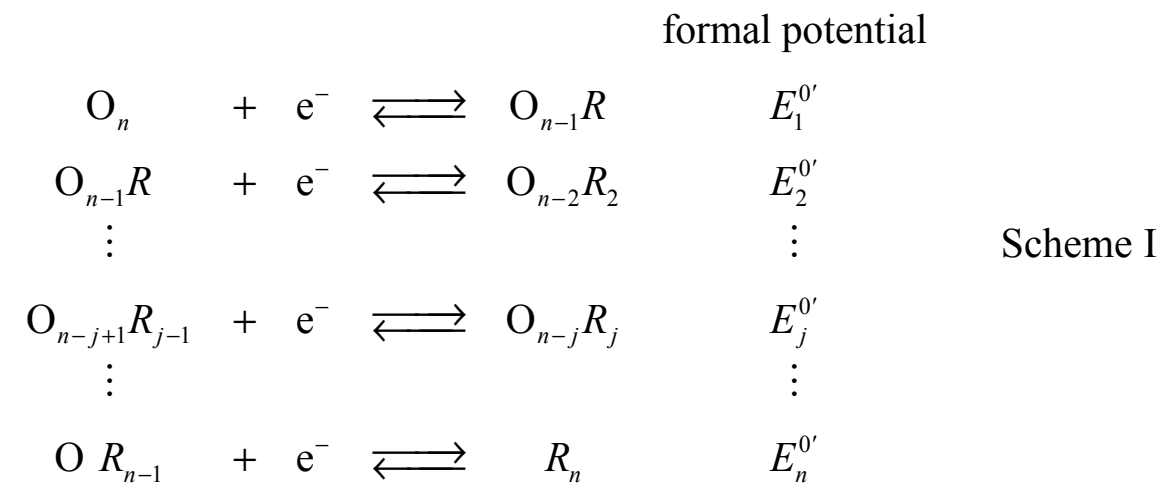

using the multipotential step technique Differential Staircase Voltammetry (DSCV) [4-8] at spherical electrodes of any size.

We assume that all reactions of the process (Scheme I) are reversible like many real and relevant systems show, namely ferrocene (one step) [9], alquil viologens (two steps) [10], natural carotenoids [11, 12], fullerenes [13-15] and other multicenter molecules such as linear polymers, supramolecular species and dendrimers (three to many steps) [1-3]. We also suppose that the diffusion coefficients of the $(n+1)$ redox states of the molecule are equal $(=D)$, in such a way that the possible $(n-1)$ reproportionation/disproportionation homogeneous electron-transfer reactions have no effect on the response in any voltammetric technique of single or multipotential step, be the diffusion field planar, spherical or even cylindrical [16].

Under these conditions, we recently derived [2] a simple explicit analytical expression for the current corresponding to the application of any pulse " $p$ " $(p \geq 1)$, in any sequence of consecutive potential steps designed, obtained at spherical electrodes of any size for molecules with multiple redox centers (interacting or not). This equation, which covers an important gap in the electrochemistry literature [2], provides the theoretical background for the analysis of the response of multicenter molecules in any multipotential step technique and in potential sweep techniques, as cyclic voltammetry, for planar electrodes, conventional sized spherical electrodes and spherical microelectrodes with which the steady state is reached. From this general equation, we derive here the particular solution for DSCV.

The DSCV technique was introduced by Scholtz et al. [4, 5] and subsequently dealt with by other authors $[6,8,7]$, as a method related to the multipulse technique Staircase Case Voltammetry (SCV) and the double pulse technique Differential Pulse Voltammetry (DPV), with the aim of obtaining a signal in the form of peaks, which is easier to evaluate than the typical signal in a SCV experiment. These authors proved that DSCV is as sensitive as DPV, although DSCV presents the clear advantage of avoiding the experimental inconvenience of the double pulse technique which, in order to reach the equilibrium every two potentials applied, needs a delay time when solid electrodes are used, or, in the case of a SMDE, a new mercury drop. However, despite the advantages, to date only simple charge transfer reactions have been studied, both theoretically and experimentally, with this technique [4-8]. 
In this work, we propose methods to determine the formal potentials and other characteristic parameters of the process for molecules with any number of strongly interacting centers when using conventional spherical electrodes and microelectrodes. For molecules with moderately interacting centers the possibility of obtaining all the formal potentials depends on the interaction degree, although the two formal potentials can always be determined for a molecule with only two redox centers. We have illustrated these results by working with two experimental systems of molecules containing two redox centers, with different interaction degrees: ethyl viologen in acetonitrile and pyrazine in aqueous acid media, and we have found an excellent agreement between the theoretical predictions and the experimental data.

\section{Experimental}

All computer programs were written in our laboratory using the program Mathcad 2001 Professional for Windows and they are available upon request. A three-electrode cell was employed in the experiments. A home-made static mercury drop electrode (SMDE) with different radius values served as working electrode. The electrode radii of the SMDE were determined by weighing a large number of drops. The counter electrode was a $\mathrm{Pt}$ wire and the reference and quasireference electrodes were a $\mathrm{Ag}|\mathrm{AgCl}| \mathrm{KCl} 1.0 \mathrm{M}$ electrode in the experiments with pyrazine and a silver wire for the ethyl viologen in acetonitrile solution.

Pyrazine, $\mathrm{NaClO}_{4}$ and $\mathrm{HClO}_{4}$ were of Merck reagent grade. Ethyl viologen, anhydrous acetonitrile and tetrabutylamonium perchlorate (TBAP) were of Aldrich reagent grade. All reagents were used without further purification. Water was bidistilled and nitrogen gas was used for deaeration. In pyrazine experiments the ionic strength has been adjusted to $1 \mathrm{M}$. In all the experiments the temperature was kept constant at $20 \pm 0.1^{\circ} \mathrm{C}$.

We used different digital noise filters of the instrument supported software in the experimental voltammetric measurements. The experimental $I_{\mathrm{SCV}} / E$ curves were smoothed by applying the moving average smoothing procedure proposed by Savitzky and Golay [17].

For the experimental curves shown in Fig.4, the background current has been corrected. The diffusion coefficient value used in the theoretical adjustment of this figure, $D=(7.5 \pm 0.1) 10^{-6} \mathrm{~cm}^{2} \mathrm{~s}^{-1}$, was previously obtained from chronoamperometric experiments and it is in good agreement with that previously reported in the literature $[18,19]$.

The zero-current points in DSCV have been calculated from linear interpolations of the curves in the differential staircase voltammograms.

\section{Theory}

In DSCV the applied potential-time waveform is the usual staircase in SCV [20, 21] (see Scheme II). In different versions of the technique, the signal is calculated as the difference between two currents measured at two different times 
at each potential step [4-6, 8], or as the difference between currents corresponding to consecutive potential steps, which can be measured once in each potential step, at the same or at different times [7, 8].

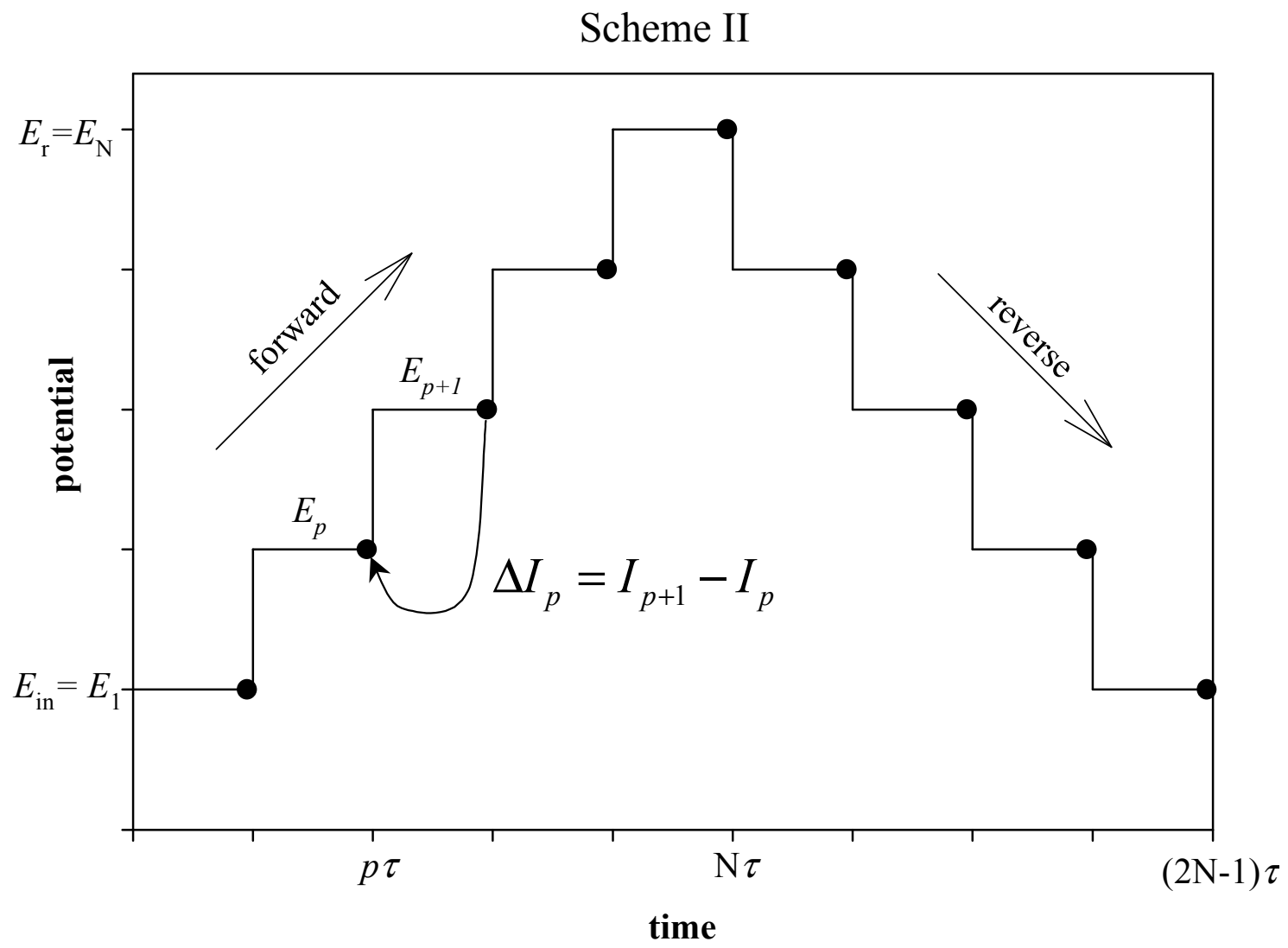

In this paper we calculate the differential signal in a parallel way to that in the double pulse technique DPV $[20,21]$, as the difference between the currents corresponding to each two consecutive pulses, $E_{p}$ and $E_{p+1}(p \geq 1)$, measured once at the end of the pulses, as shown in Scheme II.

Scheme II shows the cyclic mode of the staircase technique where all the potential pulses are of the same duration, $\tau$, and the absolute value of the difference $\Delta E=E_{k+1}-E_{k} \quad(1 \leq k \leq p-1)$ is kept constant with its sign being negative or positive according to the direction of the potential sweep. The scan is applied between the initial potential, $E_{\mathrm{in}}=E_{1}$, and the reversal potential $E_{\mathrm{r}}=E_{\mathrm{N}}$ at which the direction sweep is reversed. In these conditions, the potential sweep rate, $v$, is defined as:

$$
v=\frac{|\Delta E|}{\tau}
$$

and the response in DSCV is (see Scheme II)

$$
I_{\mathrm{DSCV}}=\Delta I_{p}=I_{p+1}-I_{p} \quad 1 \leq p \leq 2 N-2
$$


where $I_{p}$ is the current measured at the end of the $p$ th potential step, which, from Eq. (1) in Ref. [2] can be written as:

$$
\begin{aligned}
I_{\mathrm{SCV}}=I_{p} & =F A\left(\frac{D v}{\pi|\Delta E|}\right)^{1 / 2} \sum_{k=1}^{p}\left(\frac{\sum_{j=0}^{n-1}(n-j)\left[c_{j}^{k-1}\left(r_{0}\right)-c_{j}^{k}\left(r_{0}\right)\right]}{(p-k+1)^{1 / 2}}\right)+ \\
& +\frac{F A D}{r_{0}} \sum_{j=0}^{n-1}(n-j)\left[c_{j}^{0}\left(r_{0}\right)-c_{j}^{p}\left(r_{0}\right)\right]
\end{aligned}
$$

being the $I_{\mathrm{DSCV}}$ curves plotted versus $E_{p}$.

In Eq. (3), $F$ and $A$ have their usual electrochemical meanings and $c_{j}^{p}\left(r_{0}\right)$ denotes the surface concentrations of the different oxidation states of the molecule with the subscript $j(j=0,1,2, \ldots, n)$ referring to the number of reduced centers, and the superscript $p$ referring to any pulse of the sequence of potential pulses applied $(p=1,2,3, \ldots)$. The expressions for these surface concentrations are given by [2]

$$
\left.\begin{array}{c}
c_{j}^{p}\left(r_{0}\right)=\frac{c^{*} \prod_{f=j+1}^{n} J_{f}^{p}}{1+\sum_{j=1}^{n}\left(\prod_{f=j}^{n} J_{f}^{p}\right)} ; \quad j=0,1, \ldots, n-1 \\
c_{n}^{p}\left(r_{0}\right)=\frac{c^{*}}{1+\sum_{j=1}^{n}\left(\prod_{f=j}^{n} J_{f}^{p}\right)}
\end{array}\right\}
$$

where the functions $J_{j}^{p}(p \geq 1)$ depend on the potential in the way

$$
J_{j}^{p}=\exp \left(\frac{F\left(E_{p}-E_{j}^{0^{\prime}}\right)}{R T}\right) \quad(j=1,2, \ldots, n)
$$

In this paper we suppose that only the higher oxidation state, $\mathrm{O}_{n}$, of the molecule is initially present in the solution with a concentration $c^{*}$, and thus, in Eq. (3) it holds that $c_{j}^{0}\left(r_{0}\right)=0$ for $j \neq 0$ and $c_{0}^{0}\left(r_{0}\right)=c^{*}$.

Eq. (3), which has been obtained under the conditions of reversible charge transfer reactions and equal diffusion coefficients for all species participating in the process, is valid for any value of $\Delta E$ in the staircase, for any number of redox centers in the molecule, and any degree of interaction between them. 


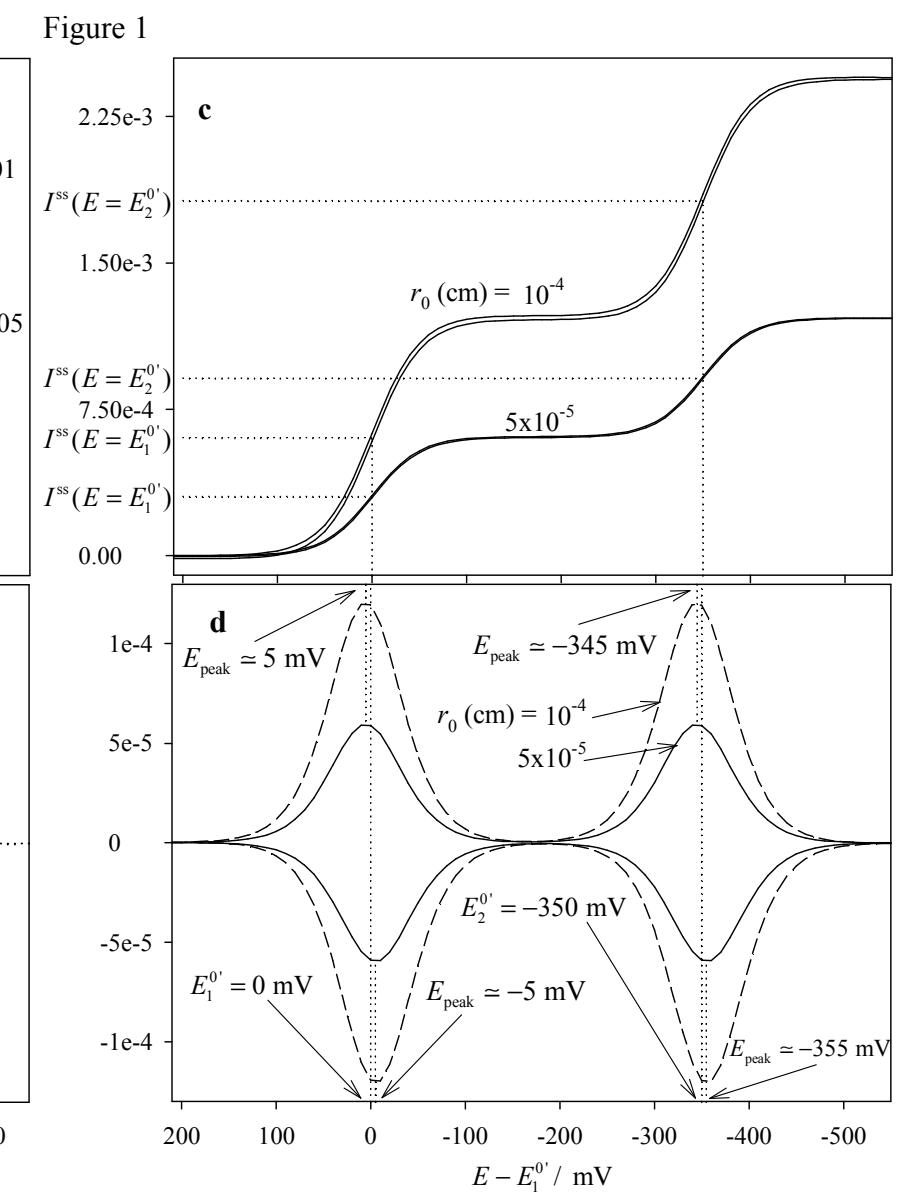

Figure 1. Influence of electrode radius, $r_{0}$, on the $I_{\mathrm{SCV}} / E$ curves [Figs. a and c, Eq. (3)], and on the $I_{\mathrm{DSCV}} / E$ curves (Figs. b and d, Eq.(2)), for a molecule containing two strongly interacting redox centers $\left(\Delta E^{0^{\prime}}\left(=E_{2}^{0^{\prime}}-E_{1}^{0^{\prime}}\right)=-350 \mathrm{mV}\right) .|\Delta E|=10 \mathrm{mV}, \quad v=100 \mathrm{mV} / \mathrm{s}, c^{*}=1 \mathrm{mM}$, $D=10^{-5} \mathrm{~cm}^{2} \mathrm{~s}^{-1}, T=298 \mathrm{~K}$. The values of $r_{0}$, in $\mathrm{cm}$, are given on the curves. 
The equation is also valid for spherical electrodes of any size, from planar to ultramicrospherical electrodes, which is of great interest, since mercury electrodes such as the SMDE are widely used in the study of multicenter molecules [13]. Note that current in Eq. (3) has been expressed as the sum of two contributions with different temporal and radial dependences. Indeed, whereas the first contribution (planar contribution) depends on time and does not depend on the electrode radius, the second one (radial contribution) is independent of time but dependent on the electrode radius (this is the current obtained for an ultramicrospherical electrode). This different temporal dependence reflects in the fact that planar contribution depends on all the potentials applied up to the potential pulse $p$, and radial contribution depends only on the actual potential step applied, $E_{p}[2]$.

\section{Results and discussion}

Fig. 1 shows the influence of the electrode radius on the SCV response (Figs. 1(a) and 1(c)) and on the corresponding DSCV curves (Figs. 1(b) and 1(d)) for a molecule with two strongly interacting redox centers $\left(\Delta E^{0^{\prime}}\left(=E_{2}^{0^{\prime}}-E_{1}^{0^{\prime}}\right)=-350 \mathrm{mV}\right)$, for which two well separated steps are observed [2]. The curves have been obtained with $|\Delta E|=10 \mathrm{mV}$ and $v=100 \mathrm{mV} / \mathrm{s}$ at two conventional spherical electrodes of different radii, $r_{0}$, (Figs. 1(a) and 1(b)) and at two microelectrodes with radii $r_{0}=10^{-4} \mathrm{~cm}$ and $r_{0}=5 \times 10^{-5} \mathrm{~cm}$ (Figs. 1(c) and 1(d)). Note that, for very small electrodes, the stationary curves corresponding to the radial contribution in Eq. (3) are obtained (Fig. 1(c)). In these conditions, for $r_{0} \rightarrow 0$, the DSCV response (Fig. 1(d)) is identical to that in the double pulse technique DPV (as indicated by the same current peaks for the two steps [22, 16]). Thus, the formal potentials for both steps, $E_{1}^{0^{\prime}}$ and $E_{2}^{0^{\prime}}$, can easily be obtained from the peak potentials, since:

$$
\begin{aligned}
& E_{1}^{0^{\prime}}=E_{\text {first peak }} \mp \frac{\Delta E}{2} \\
& E_{2}^{0^{\prime}}=E_{\text {second peak }} \mp \frac{\Delta E}{2}
\end{aligned}
$$

where the upper and lower signs in Eqs. (6) and (7) refer to the cathodic and anodic peaks.

The similarity between DSCV and DPV was already remarked and proved by other authors $[4,5]$, who found that DSCV is as sensitive as DPV and moreover, since in both techniques the signal is obtained by a difference of currents, the background current is significantly suppressed. Nevertheless, DSCV presents the clear advantage of avoiding the experimental inconvenience of the double pulse technique which, in order to reach the equilibrium every two potentials applied, needs a delay time when solid electrodes are used, or, in the case of a SMDE, a new mercury drop. 
Once the formal potentials are known, we can determine other characteristic parameters of the system, such as $D$ and $c^{*}$, from the stationary response shown in Fig. 1(c). Indeed, from Eq. (3) (radial contribution) and taking into account

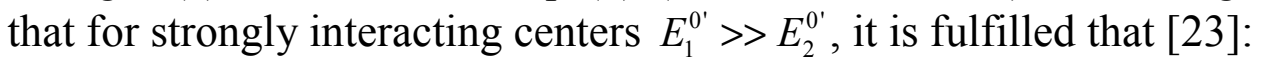

$$
\begin{aligned}
& I^{\mathrm{ss}}\left(E=E_{1}^{0^{\prime}}\right)=\frac{F A D c^{*}}{r_{0}} \frac{1}{2} \\
& I^{s s}\left(E=E_{2}^{0^{\prime}}\right)=\frac{F A D c^{*}}{r_{0}} \frac{3}{2}
\end{aligned}
$$

For conventional spherical electrodes the typical curves in DSCV are those shown in Fig. 1(b), for which the Eqs. (6) and (7) are not fulfilled. Nevertheless, the formal potentials can still be easily obtained from the zero-current points that appear in the response (see Fig. 1(b)) for which it is fulfilled that:

$$
\begin{aligned}
& E_{1}^{0^{\prime}}=\frac{E^{\prime \prime} 1^{\prime \prime}+E^{\prime \prime 2}}{2} \\
& E_{2}^{0^{\prime}}=\frac{E^{\prime \prime 5^{\prime \prime}}+E^{\prime \prime} 6^{\prime \prime}}{2}
\end{aligned}
$$

and also:

$$
\frac{E_{1}^{0^{\prime}}+E_{2}^{0^{\prime}}}{2}=\frac{E^{\prime \prime 3^{\prime \prime}}+E^{\prime \prime} 4^{\prime \prime}}{2}
$$

Thus, the formal potentials can be determined from a linear interpolation of the central zone of the curve around each zero-current point and by applying Eqs. (10) and (11). These zero-current points depend on the electrode radius (see Fig. 1(b)), but Eqs. (10)-(12) hold for any radius of a conventional spherical electrode.

In this case of strongly interacting centers, the results in Fig. 1 can be extended to molecules with any number of redox centers.

In order to corroborate the theoretical predictions, in Fig. 2 we have plotted the experimental curves obtained for different values of the radius of a mercury electrode in SCV (Fig. 2(a)) and in DSCV (Fig. 2(b)) for the ethyl viologen in acetonitrile system. In Fig. 2(a) the typical curves corresponding to wellseparated steps are observed and the values of the formal potentials can be determined from the curves in Fig. 2(b), as explained for Fig. 1(b). Thus, the values obtained are $E_{1}^{0^{\prime}}=-0.4372 \pm 0.001 \mathrm{~V}$ and $E_{2}^{0^{\prime}}=-0.8566 \pm 0.001 \mathrm{~V}$ $\left(\Delta E^{0^{\prime}}=-0.4194 \pm 0.002 \mathrm{~V}\right)$. We have also determined the semisum of $E^{\prime 3 "}$ and 
$E^{\prime 4 "}$, obtaining $\frac{E^{\prime 3 "}+E^{\prime 4 "}}{2}=-0.6460 \pm 0.001 \mathrm{~V}$, which practically coincides with the semisum of formal potentials $\frac{E_{1}^{0^{\prime}}+E_{2}^{0^{\prime}}}{2}=-0.6470 \pm 0.001 \mathrm{~V}$.

Figure 2

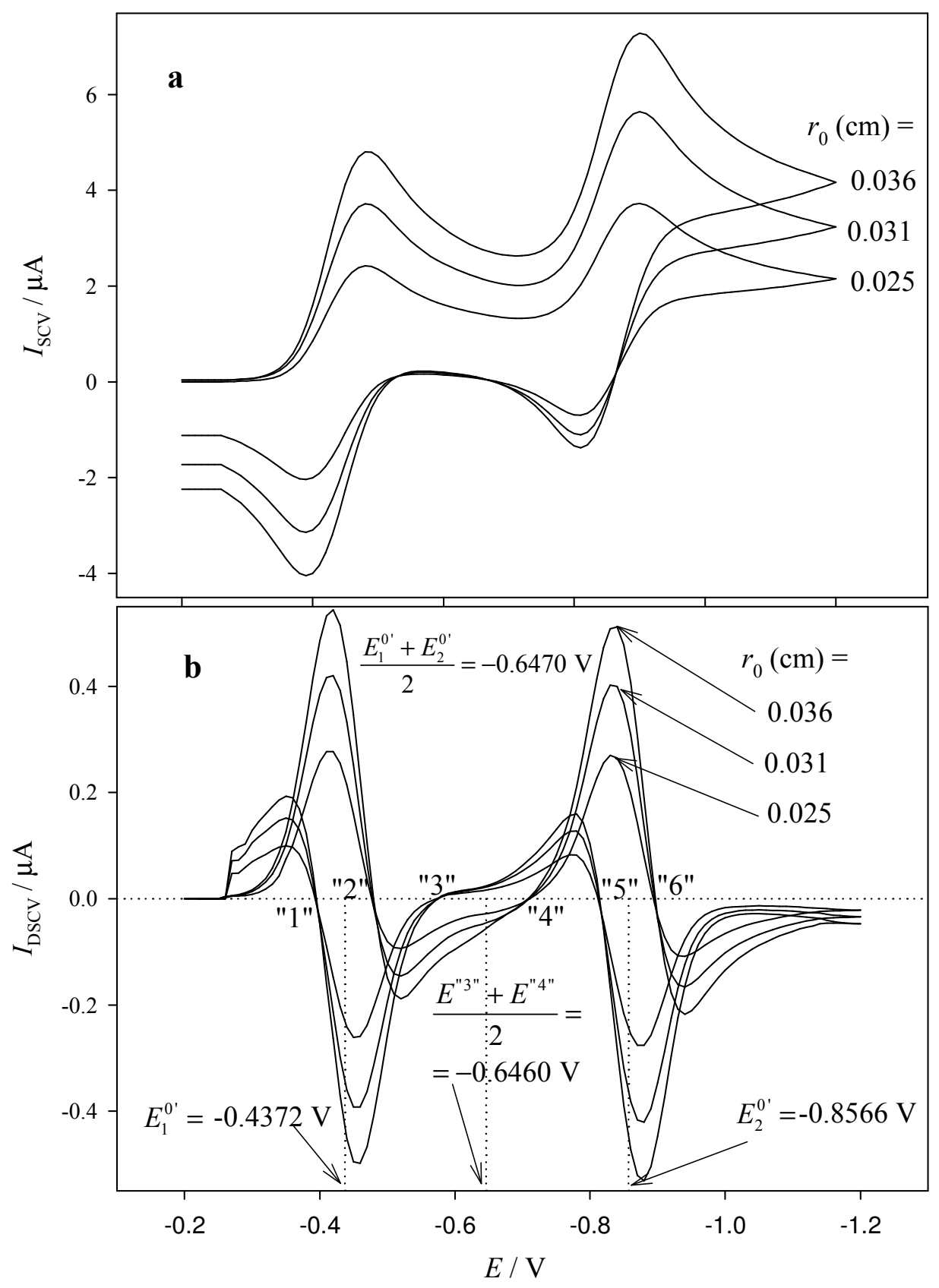

Figure 2. Influence of the radius of a SMDE, $r_{0}$, on the experimental $I_{\mathrm{SCV}} / E$ curves (Fig. a), and on the $I_{\mathrm{DSCV}} / E$ curves (Fig. b) obtained for ethyl viologen $1 \mathrm{mM}+$ TBAP $0.1 \mathrm{M}$ in acetonitrile. $|\Delta E|=10 \mathrm{mV}, v=0.1 \mathrm{~V} / \mathrm{s}, T=293 \mathrm{~K}$. The values of $r_{0}$, in $\mathrm{cm}$, are given on the curves. 
Fig. 3 is the equivalent to Fig. 1 but for a molecule with two moderately interacting redox centers. This case of intermediate interactions corresponds to not well separated electrochemical steps $\left(\Delta E^{0^{\prime}}\left(=E_{2}^{0^{\prime}}-E_{1}^{0^{\prime}}\right)=-125 \mathrm{mV}\right)$ and thus overlapped waves are observed $[22,16,2]$. Nevertheless, it can be noticed that while the response in SCV (Figs. 3(a) and 3(c)) hardly shows the presence of two steps, two peaks clearly appear in the corresponding curves in DSCV (Figs. 3(b) and 3(d)). For the small radius used in Fig. 3(d) the response is practically coincident to that in $\operatorname{DPV}[22,16]$, and therefore the formal potentials can be obtained from the peak potentials and using Eqs. (6) and (7), with an error of less than $3 \mathrm{mV}$. Eqs. (8) and (9), which refer to the stationary current in Fig. 3(c), also hold in this case, although these equations have been obtained under the condition of well-separated steps. Therefore, the use of microelectrodes has the effect of separating the steps. This behaviour is observed if $\Delta E^{0^{\prime}} \leq-125 \mathrm{mV}$, for any number of redox centers. For higher values of $\Delta E^{0^{\prime}}$, Eqs. (6) and (7) cannot be applied to determine the formal potential, regardless of how much the electrode radius decreases.

However, when conventional spherical electrodes are used, it is possible to obtain $E_{1}^{0^{\prime}}$ and $E_{2}^{0^{\prime}}$ from the response in DSCV (Fig. 3(b)) for any interaction degree between the centers (any value of $\Delta E^{0^{\prime}}$ ). In this case it is fulfilled that (see Fig. 3(b)):

$$
\frac{E_{1}^{0^{\prime}}+E_{2}^{0^{\prime}}}{2}=\frac{E^{\prime \prime l^{\prime \prime}}+E^{\prime \prime 2}}{2}
$$

and hence, the formal potentials can be determined by adjusting the experimental response and a theoretical curve obtained from Eq. (2) with the appropriate value of $\Delta E^{0^{\prime}}\left(=E_{2}^{0^{\prime}}-E_{1}^{0^{\prime}}\right)$, and using Eq. (13), as is illustrated in Fig. 4.

In Fig. 4 we have plotted the experimental curves (dotted lines) obtained at two values of the radius of a mercury electrode, $r_{0}$, in SCV (Fig. 4(a)) and in DSCV (Fig. 4(b)) for the pyrazine in aqueous acid media system. In solid lines appear the corresponding theoretical curves, which have been calculated for $\Delta E^{0^{\prime}}\left(=E_{2}^{0^{\prime}}-E_{1}^{0^{\prime}}\right)=-0.126 \mathrm{~V}$. As can be seen, an excellent adjustment between theoretical and experimental curves is obtained for this value. In these conditions the semisum of potentials " 1 " and "2", which was obtained by a linear interpolation of the zone around the zero-current point of the experimental curves, has the value $\frac{E^{\prime 1 "}+E^{\prime 2 "}}{2}=-0.327 \pm 0.001 \mathrm{~V}$. Thus, from this value and $\Delta E^{0^{\prime}}$ we have obtained for $E_{1}^{0^{\prime}}$ and $E_{2}^{0^{\prime}}$ the values $E_{1}^{0^{\prime}}=-0.264 \pm 0.001 \mathrm{~V}$ and $E_{2}^{0^{\prime}}=-0.390 \pm 0.001 \mathrm{mV}$. 

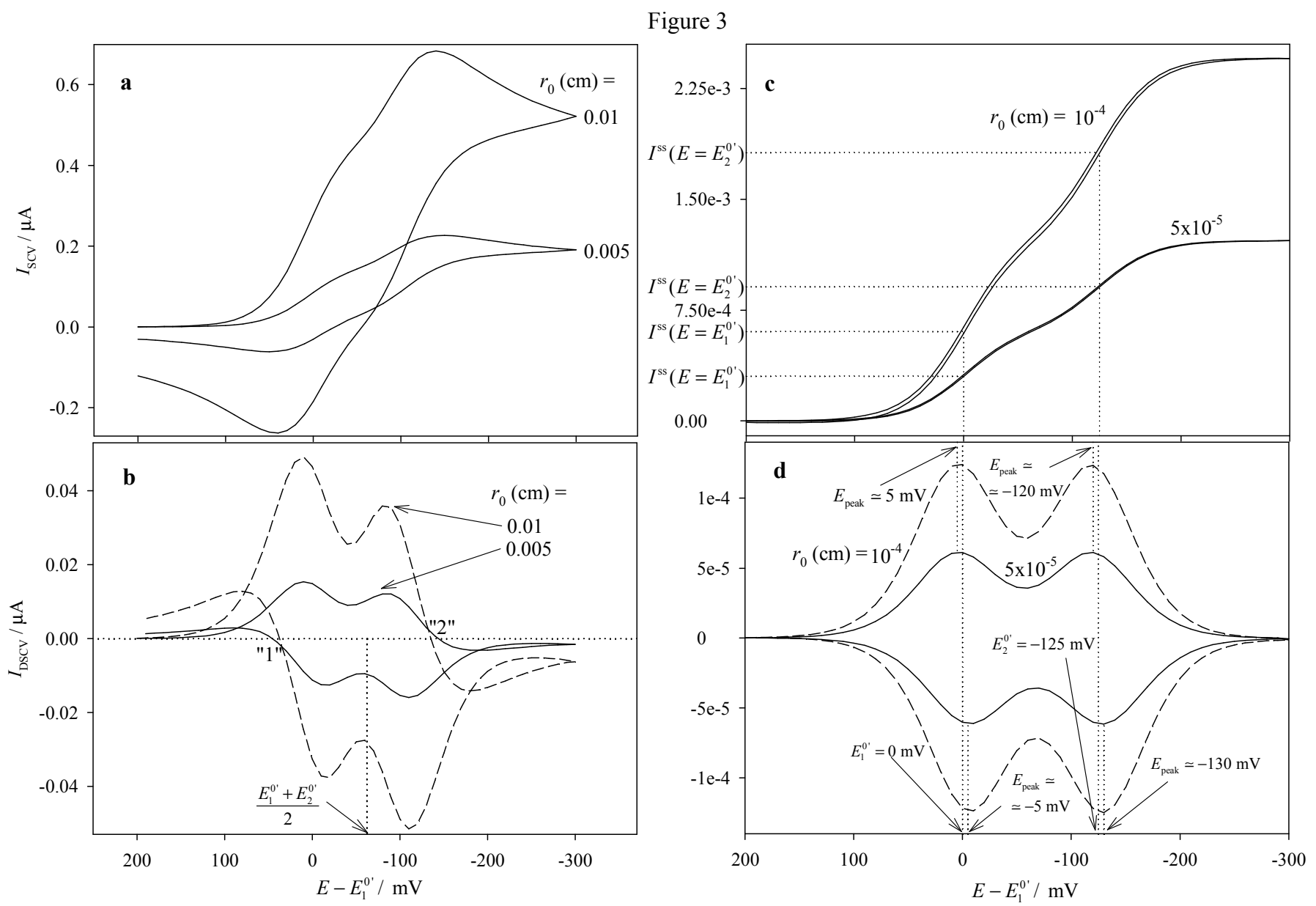

Figure 3. Influence of electrode radius, $r_{0}$, on the $I_{\mathrm{SCV}} / E$ curves (Figs. a and c, Eq. (3)), and on the $I_{\mathrm{DSCV}} / E$ curves (Figs. b and d, Eq. (2)), for a molecule containing two partially interacting redox centers $\left(\Delta E^{0^{\prime}}\left(=E_{2}^{0^{\prime}}-E_{1}^{0^{\prime}}\right)=-125 \mathrm{mV}\right)$. The values of $r_{0}$, in cm, are given on the curves. Other conditions as in Fig. 1. 
Figure 4

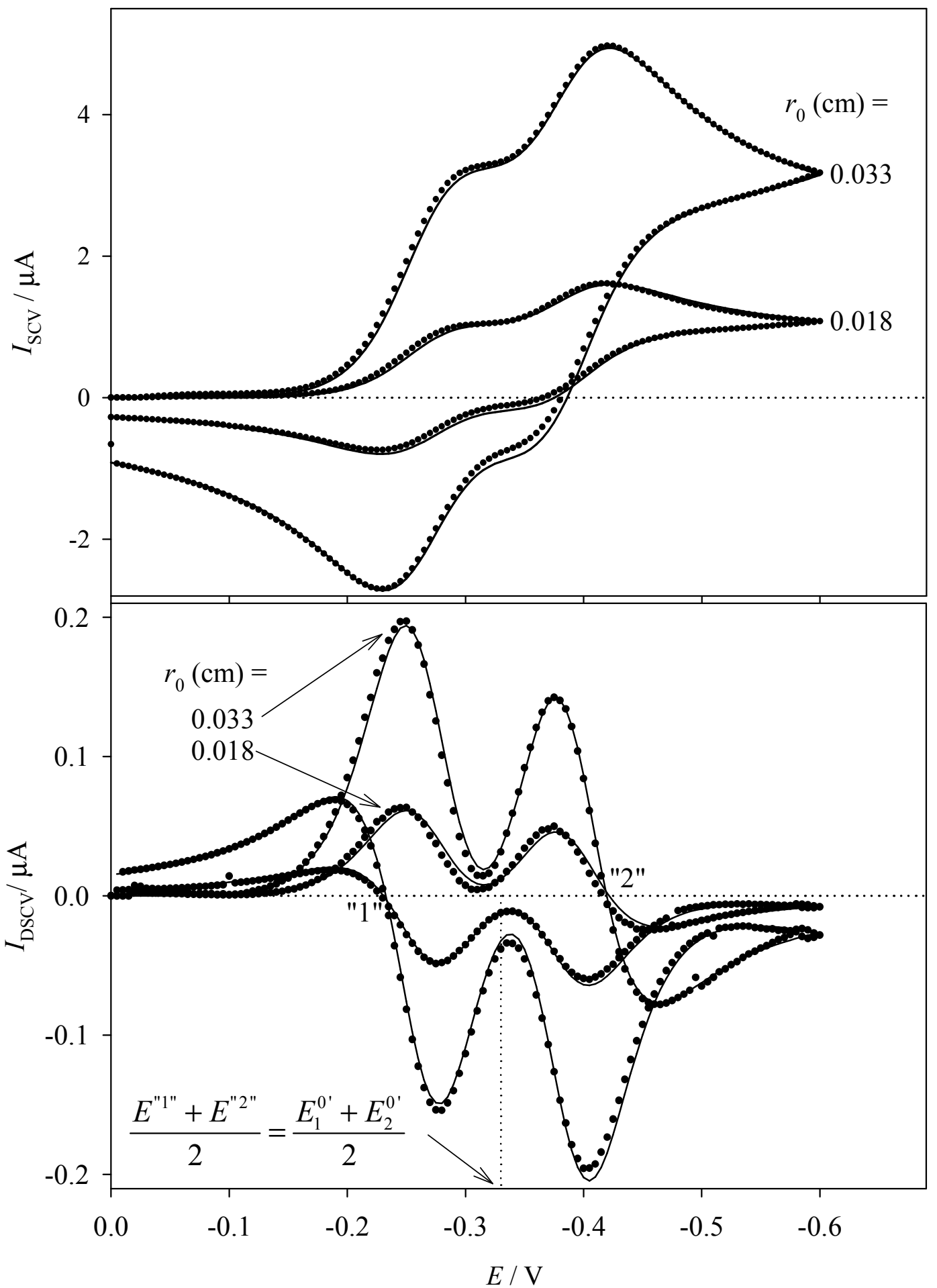

Figure 4. Comparison between experimental curves for pyrazine $1.0 \mathrm{mM}$ at $\mathrm{pH}=0.35$ in $\mathrm{HClO}_{4}+\mathrm{NaClO}_{4}$ (dotted lines) and theoretical curves for the case of a molecule with two redox centers with $\Delta E^{0^{\prime}}=-0.126 \mathrm{~V}$ (solid lines), obtained for two values of the radius of a SMDE, $r_{0}$. (a) Curves in SCV (Eq. (3) with $n=2$ ), (b) Curves in DSCV (Eq. (2) with $n=2) . \quad|\Delta E|=0.005 \mathrm{~V}, \quad v=0.5 \mathrm{~V} / \mathrm{s}, \quad T=293 \mathrm{~K}$, $D=0.7510^{-5} \mathrm{~cm} \mathrm{~s}^{-1}$. The values of $r_{0}$, in $\mathrm{cm}$, are given on the curves. 

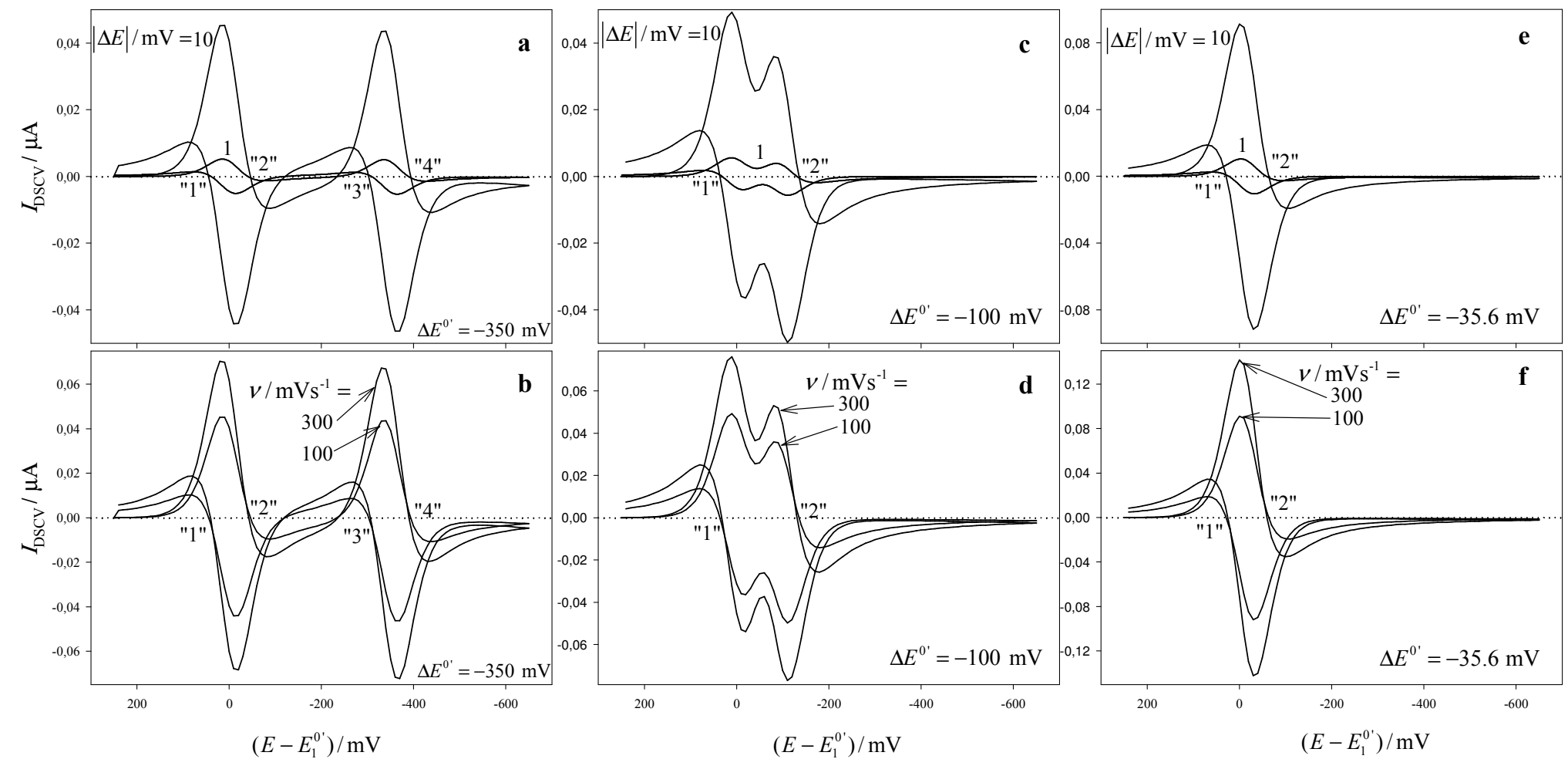

Figure 5

Figure 5. Influence of pulse amplitude, $|\Delta E|$ (Figs. a, c and e) and sweep rate, $v$ (Figs. B, $\mathrm{d}$ and f) on the $I_{\mathrm{DSCV}} / E$ curves (Eq. (2)), for molecules with two redox centers with different interaction degrees. Figs. a and b: strongly interacting centers, $\Delta E^{0^{\prime}}=-350 \mathrm{mV}$; Figs. $\mathrm{c}$ and d: moderately interacting centers, $\Delta E^{0^{\prime}}=-100 \mathrm{mV}$; Figs. e and f: noninteracting centers, $\Delta E^{0^{\prime}}=-35.6 \mathrm{mV}[24,2] . r_{0}=0.01 \mathrm{~cm}$. The values of $|\Delta E|$, in $\mathrm{mV}$, and $v$, in $\mathrm{mV} / \mathrm{s}$, are given on the curves. Other conditions as in Fig. 1 . 
Fig. 5 shows the influence of the pulse amplitude, $|\Delta E|$ (Figs. (a), (c) and (e)), and sweep rate, $v$ (Figs. (b), (d) and (f)), on the $I_{\mathrm{DSCV}} / E$ (Eq. (2)), obtained for a molecule with two redox centers with different interaction degrees at a spherical electrode of radius $r_{0}=0.01 \mathrm{~cm}$. In Figs. (a) and (b) the case of two strongly interacting centers (see Figs. 1 and 2) is considered. In Figs. (c) and (d) the interaction between the centers is smaller (moderately interacting centers), as in Figs. 3 and 4. Finally, Figs. (e) and (f) have been obtained for a molecule with two noninteracting redox centers $\left(\Delta E^{0^{\prime}}\left(=E_{2}^{0^{\prime}}-E_{1}^{0^{\prime}}\right)=-35.6 \mathrm{mV}\right)$, with the current in this case being twice that obtained for a single reversible one-electron transfer $[24,2]$.

In this figure it is observed that varying the pulse amplitude or the sweep rate has a similar effect on the curves as changing the electrode radius (see Figs. 1(b) and 3(b)), in such a way that, when using conventional spherical electrodes, the formal potentials can be obtained from the zero-current points shown in these figures, as explained for Figs. 1(b) and 3(b), for any value of $|\Delta E|$ and $v$.

\section{Conclusions}

- In this work we have applied the multipotential step technique Differential Staircase Voltammetry (DSCV) to the study of molecules with multiple redox centers, interacting or not.

- The equation used in this paper for the response in DSCV has been obtained from the theory developed in Ref. [2] for any multipotential pulse technique. This equation, which has been obtained under the conditions of reversible charge transfer reactions and equal diffusion coefficients for all species participating in the process, is valid for any value of $\Delta E$ in the staircase, for any number of redox centers in the molecule, and any degree of interaction between them, and also for spherical electrodes of any size, from planar to ultramicrospherical electrodes.

- When microelectrodes are used, the DSCV response is identical to that in Differential Pulse Voltammetry (DPV), although DSCV presents the clear advantage of avoiding the experimental inconvenience of the double pulse technique which, in order to reach the equilibrium every two potentials applied, needs a delay time when solid electrodes are used, or, in the case of a SMDE, a new mercury drop.

- We propose methods to determine the formal potentials and other characteristic parameters of the process for molecules with any number of strongly interacting centers when using conventional spherical electrodes and microelectrodes.

- For molecules with moderately interacting centers the use of microelectrodes has the practical effect of separating the electrochemical steps. In this case, the possibility of obtaining all the formal potentials depends on the interaction degree, although the two formal potentials can always be determined for a molecule with only two redox centers. 
- We have corroborated the theoretical results by working with two experimental systems of molecules containing two redox centers, with different interaction degrees: ethyl viologen in acetonitrile and pyrazine in aqueous acid media, and we have evaluated the formal potentials using DSCV.

\section{Acknowledgements}

The authors greatly appreciate the financial support provided by the Dirección General de Investigación Científica y Técnica (Projects Number BQU200304172 and CTQ2006-12552/BQU) and by the Fundación SENECA (Expedients number $\mathrm{PB} / 53 / \mathrm{FS} / 02$ and 03079/PI/05). M. M. M. thanks the Fundación CAJAMURCIA for the postdoctoral grant received.

\section{References}

1. A.J. Bard, Nature 374 (1995) 13.

2. A. Molina, C. Serna, M. López-Tenés, M.M. Moreno, J. Electroanal. Chem. 576 (2005) 9.

3. B.K. Roland, W.H. Flora, H.D. Selby, N.R. Armstrong, Z. Zheng, J. Am. Chem. Soc. 128 (2006) 6620.

4. F. Scholz, L. Nitschke, G. Henrion, Fresenius Z. Anal. Chem. 332 (1988) 805.

5. F. Scholz, M. Draheim, G. Henrion, Fresenius J. Anal. Chem. 336 (1990) 136.

6. M. Lovric, Croatica Chemica Acta 68(2) (1995) 335.

7. D. Krulic, N. Fatouros, M.M. ElBelamachi, J. Electroanal. Chem. 385 (1995) 33.

8. J.-S. Yu, Z.-X. Zhang, J. Electroanal. Chem. 427 (1997) 7.

9. J. González, A. Molina, M. López-Tenés, C. Serna, J. Electrochem. Soc. 147 (2000) 3429.

10. C.L. Bird, A.T. Kuhn, Chem. Soc. Rev. 10 (1981) 49.

11. K.M. Kadish, C. Araullo, G.B. Maiya, D. Sazou, J.M. Barbe, R. Guilard, Inorg. Chem. 28 (1989) 2528.

12. D. Liu, Y. Gao, L.D. Kispert, J. Electroanal. Chem. 488 (2000) 140.

13. G. Diao, Z. Zhang, J. Electroanal. Chem. 414 (1996) 177.

14. G. Diao, Z. Zhang, J. Electroanal. Chem. 429 (1997) 67.

15. E. Dietel, A. Hirsch, J. Zhou, A. Rieker, J. Chem. Soc., Perkin Trans. 2 (1998) 1357.

16. C. Serna, A. Molina, M.M. Moreno, M. López-Tenés, J. Electroanal. Chem. 546 (2003) 97.

17. A. Savitzky, M.J.E. Golay, Anal. Chem. 36 (1964) 1627.

18. A. Molina, M.M. Moreno, M. López-Tenés, C. Serna, Electrochem. Commun. 4 (2002) 457.

19. J. Swartz, F.C. Anson, J. Electroanal. Chem. 114 (1980) 117.

20. Z. Galus, in: (Ed.), Fundamentals of Electrochemical Analysis, Ellis Horwood, Chichester, 1994, Chapter 11 
21. A.J. Bard, L.R. Faulkner, in: (Ed.), Electrochemical Methods. Fundamental and Applications, Wiley, New York, 2001.

22. M. López-Tenés, M.M. Moreno, C. Serna, A. Molina, J. Electroanal. Chem. 528 (2002) 159.

23. M. López-Tenés, A. Molina, C. Serna, M.M. Moreno, J. González, J. Electroanal. Chem. (2007), doi:10.1016/j.jelechem.2007.02.011.

24. J.B. Flanagan, S. Margel, A.J. Bard, F.C. Anson, J. Am. Chem. Soc. 100 (1978) 4248. 